\title{
Real-time atrial wall imaging during radiofrequency ablation in a porcine model
}

\author{
Mathieu Granier, MD, MSc, ${ }^{*}$ Pierre François Winum, MD, ${ }^{*}$ Mireille Granier, MD, ${ }^{\dagger}$ \\ Pierre Liaud, MSc, ${ }^{\ddagger}$ Guillaume Cayla, MD, PhD, ${ }^{*} \S$ Patrick Messner, MD, PhD, ${ }^{*} \S$ \\ Jean-Luc Pasquie, MD, PhD, $\|$ II Iris Schuster, MD, PhD* ${ }^{*}$ \\ From the ${ }^{*}$ Cardiology Department, Nimes University Hospital, Nimes, France, ${ }^{\dagger}$ Pathology Department, \\ Montpellier University Hospital, Montpellier, France, ${ }^{\star}$ EA 2992: Dysfunction of Vascular Interfaces, \\ Faculty of Medicine, Montpellier University, Nimes, France, ${ }^{\S}$ Montpellier University, Nimes, France, \\ "Cardiology Department, Montpellier University Hospital and "IINSERM U1046 Montpellier University, \\ Montpellier,France.
}

BACKGROUND Real-time monitoring of radiofrequency (RF) ablation remains challenging.

OBJECTIVE We used intravascular ultrasound (IVUS) to describe atrial wall changes during RF ablation and to assess the extent of RF-induced lesions.

METHODS In 9 piglets, RF and IVUS catheters were coupled and introduced into the right atrium. RF applications were performed along the intercaval line. Corresponding IVUS images were analyzed. Wall thickness was correlated with electrogram (EGM) changes $(n=9)$ and histology $(n=5)$.

RESULTS There were 66 RF applications performed in 57 sites. IVUS provided real-time imaging of the atrial wall during RF application in all but 2 sites. IVUS demonstrated significant $(>20 \%)$ and immediate increase in atrial wall thickness in $71.4 \%$ of RF applications. It showed epicardial or intramyocardial effusion in $30 \%$ of cases, 2 steam pops, 1 intramural hematoma, and 1 thrombus. EGM amplitude decreased and thickness increased after RF application than at baseline $(2.20 \pm 1.11$ to $0.99 \pm 0.62 \mathrm{mV}$ and $1.34 \pm 0.53$ to $1.93 \pm 0.80 \mathrm{~mm}$, respectively; $P<.001$ for each).

\section{Introduction}

Radiofrequency (RF) energy is widely used since the beginning of the 1990 s to treat arrhythmias. ${ }^{1,2}$ However, little is known about the real-time in vivo effect of RF ablation on the atrial wall. Monitoring of RF ablation is limited to indirectly acquired variables such as temperature, impedance, or electrical signal, ${ }^{3-5}$ and uncertainty about the

This work was funded by EA 2992: Dysfunction of Vascular Interfaces, Faculty of Medicine, Montpellier I University, Nimes, France. This work was supported by Boston Scientific and St Jude Medical, which provided logistical support. Address reprint requests and correspondence: Dr Mathieu Granier, Service de Cardiologie, Hopital Carremeau, 1 place Robert Debré, 30029 Nimes Cedex 9, France. E-mail address: mathieu.granier@chu-nimes.fr.
However, EGM and thickness changes were poorly correlated ( $r=$ 0.43; $P<.05)$. Histologically and echographically measured thicknesses were correlated $(r=0.71 ; P=.004)$, but echographic thickness change was not related to histological lesion transmurality.

CONCLUSION An IVUS probe coupled to an RF catheter can provide relevant real-time imaging of the atrial wall during ablation. Although thickness change does not appear as a good predictor of the transmural extent, direct visualization and monitoring of RF application may provide new information to guide and secure RF ablation.

KEYWORDS Radiofrequency ablation; RF ablation; Transmural extent of RF lesions; Radiofrequency ablation in a porcine model; IVUS imaging of atrial wall; RF lesion extent; Transmural lesion extent; Atrial fibrillation

ABBREVIATIONS EGM = electrogram; IVUS = intravascular ultrasound; $\mathbf{R A}=$ right atrium; $\mathbf{R F}=$ radiofrequency

complete achievement of a transmural lesion may impair RF treatment efficacy. ${ }^{6}$

In contrast, complications of RF ablation remain most of the time unpredictable owing to the lack of reliable information about morphological atrial wall tissue changes. Recently, new catheters providing contact force information demonstrated their ability to improve quality and reliability of RF. ${ }^{7-9}$ Their use emphasized the transmural extent of the lesion as a critical issue for procedural success. ${ }^{10}$

Several attempts have been made to provide additional information on functional and morphological RF-induced changes. ${ }^{11-16}$ Photoacoustic imaging has been used in vitro with encouraging results. ${ }^{13}$ Magnetic resonance imaging is limited by spatial resolution and the need of magnetic resonance imaging-compatible equipment. ${ }^{15}$ Optical coherence tomography is limited by depth resolution, ${ }^{16}$ and 
acoustic radiation force impulse is still limited in vivo by motion artifacts. ${ }^{12}$ One study used a laboratory-made Mmode catheter, ${ }^{11}$ and we found no report of the use of clinically available catheters and 2-dimensional echographic imaging.

Ultrasound imaging has been used for years during ablation procedures to monitor catheter position, ${ }^{17,18}$ to help electroanatomical reconstruction, ${ }^{19}$ or to visualize anatomical changes, ${ }^{20,21}$ but this was obtained with intracardiac or transesophageal probes that did not offer sufficiently high-definition imaging of the atrial wall throughout RF application.

Intravascular ultrasound (IVUS) imaging use high-frequency (40-MHz) small-diameter (3.5-F) probes and is routinely used for interventional cardiology to perform virtual histology of atherosclerotic plaque and to assess angioplasty results. ${ }^{22}$

We hypothesized that an IVUS probe could be coupled to an RF ablation catheter to assess in real time the morphological changes in the atrial wall during RF application in a porcine model. Our first aim was to describe the RF effects on the atrial wall, thus evaluating the ability of highresolution B-mode ultrasonography to guide and secure RF ablation. Our secondary goal was to investigate whether thickness changes in the atrial wall could be relevant to assess the transmural extent of RF lesions as assessed by electrogram (EGM) amplitude changes and histology.

\section{Methods}

\section{Animals and experimental protocol}

The experimental protocol was approved by the regional ethics committee for animal research (approval no. CEEA-LR-12065). nine large white piglets (weighing $20-30 \mathrm{~kg}$ ) were anesthetized by intramuscular injection of ketamine $(10 \mathrm{mg} / \mathrm{kg})$ and midazolam $(1 \mathrm{mg} / \mathrm{kg})$, intubated, and mechanically ventilated. Sedation was maintained with propofol $(4 \mathrm{mg} / \mathrm{kg}$ bolus, followed by $8 \mathrm{mg} / \mathrm{kg} / \mathrm{h}$ infusion) and cisatracurium $(0.25 \mathrm{mg} / \mathrm{kg}$ bolus followed by $8 \mathrm{mg} / \mathrm{kg} / \mathrm{h}$ infusion). Bilateral femoral venous puncture was performed, a 14-F long sheath was introduced through the right femoral vein, and a 6-F sheath was introduced through the left femoral vein. Unfractionated heparin (100 IU/kg) was administered before the beginning of the procedure (Figure 1).

Animals were divided into 2 groups: In group $1(n=4)$, several RF applications were performed on the posterior wall of the right atrium (RA) to search for correlations between EGM amplitude and wall thickness changes. In group $2(n=$ 5), a maximum of 4 largely separated spots of RF applications was allowed, along the intercaval line, in order to allow easy excision of the lesion for histopathology analysis and correlation with EGM and wall thickness changes (Figure 1).

\section{Electrophysiology and ablation procedure}

An 8-F diameter/5-mm-tip nonirrigated ablation catheter (Blazer II, Boston Scientific, Malborough, MA) was coupled to a 3.5-F IVUS catheter (40 MHz) (Atlantis Pro, Boston Scientific) and introduced via the 14-F sheath into the RA. A deflectable quadripolar diagnostic catheter (Xtrem, Sorin, Milan, Italy) was introduced via the 6-F sheath into the coronary sinus to monitor sinus rhythm.

Electrical signals from the 4-lead surface electrocardiogram and the distal bipoles of the catheters were processed

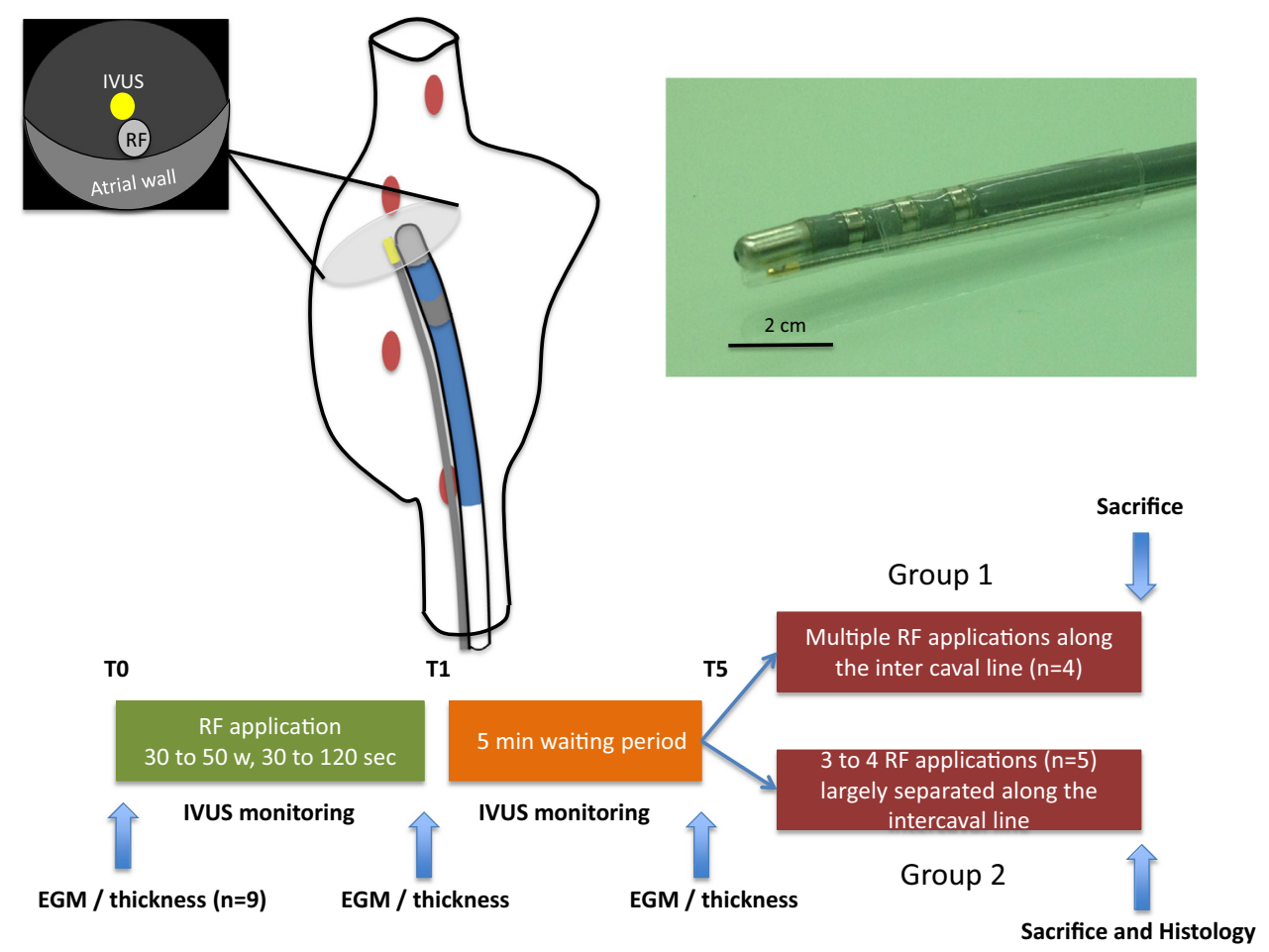

Figure 1 Experimental protocol and schematic representation of radiofrequency (RF) imaging with intravascular ultrasound (IVUS) and RF catheter coupled together. Top right: Photograph of the 2 catheters coupled together. 
with a $30-$ to $500-\mathrm{Hz}$ bandpass filter and recorded digitally (EP MedSystems, Inc, St Jude Medical, St Paul, MN). RF energy was delivered by a dedicated generator (EPT, Boston Scientific, Malborough, MA) connected to the digital recording system, allowing continuous monitoring of RF application parameters (power, impedance, and duration).

RF was applied to separate sites, distributed between the superior vena cava and the inferior vena cava, using various settings of energy and duration after ensuring stable catheter position and good image quality (power range $30-50 \mathrm{~W}$; duration 30-120 seconds; temperature cutoff $60^{\circ} \mathrm{C}$ ). To ensure good image quality, RF was applied only on plane surfaces (posterior or posteroseptal wall). Electrical amplitude, bipolar signal waveform, and cycle length were recorded immediately before and after the application of $\mathrm{RF}$ and after 5-10 minutes under the stable condition. A second or a third application at the same site could be performed if there was no dramatic change in the bipolar EGM or IVUS image, and the catheter position was stable.

\section{IVUS imaging}

IVUS imaging was performed with an i-lab station (Boston Scientific). Atlantis Pro is an IVUS 40-MHz rotational probe providing images with an axial resolution of $0.043 \mathrm{~mm}$ and a penetration depth of $6 \mathrm{~mm}$. The outer diameter of imaging window is $3.5 \mathrm{~F}$. Images were stored and manually reviewed for thickness measurement after the procedure. Runs were recorded throughout the RF application, and after 5-10 minutes a stable condition could be maintained.

\section{Assessment of RF effect on the atrial wall}

B-mode images were analyzed for changes in thickness, echogenicity, and shape of the atrial wall. Interposition of an anechoic lamina was interpreted as epicardial effusion if occurring between the epicardium and the myocardium and as intramyocardial effusion if occurring within the myocardium. A greater than $20 \%$ increase in wall thickness was interpreted as edema. A mobile hyperechogenic structure was interpreted as a mural thrombus if occurring in the atrial cavity and as an intramural thrombus if occurring in the atrial wall.

\section{Correlation between wall thickness changes and electrical or histological data}

Echographic and histological measurements of the atrial wall thickness were performed immediately adjacent to the RF catheter apposition site, perpendicularly to the endocardial plane, from the endocardium to the epicardium. In vivo echographic measurements were performed before and after RF application and repeated 5 minutes later if the catheter and IVUS images were stable. Transmural RF ablation was defined as a greater than $80 \%$ decrease in EGM amplitude. ${ }^{23,24}$

\section{Histology}

Animals were euthanized immediately after the procedure, and their heart was explanted and rinsed. Areas of RF lesions were excised and fixed in 10\% formalin. They were then cut perpendicularly to the surface in the central part of the lesion, paraffin embedded, and serial $5-\mu \mathrm{m}$ sections were obtained with a motorized microtome. Sections were stained with Masson's trichrome and hematein-eosin. Histological analysis was performed with a light microscope (Leitz Dialux, Leica microsystem, Wetzlar, Germany). Photographs and measurements were taken with Labscope software (Zeiss). RF lesions were identified as color changes with Masson's trichrome from red (no RF lesion) to blue (RF lesion). The site of contact of the RF catheter was shown by a depression in the endocardial surface and/or a fibrin deposit. The transmural extent of RF lesions was assessed from the presence of blue stain throughout the atrial wall facing the endocardial area of contact with the RF catheter. ${ }^{24}$

\section{Statistics}

Statistical analysis was achieved with Prism V6 software (GraphPad, La Jolla, CA). Data are expressed as mean \pm SD. Quantitative variables before and after RF application were compared using a 2-tailed paired $t$ test. The Mann-Whitney $U$ test was used to compare histological and electrical data on the transmural extent of lesions. The Pearson coefficient was used to assess correlations between variables. The results were considered to be significant if the $P$ value was $<.05$.

\section{Results}

Nine Large White piglets were included. Five of them underwent histological and pathological examination (group 2). A total of $66 \mathrm{RF}$ applications were performed in 57 sites in the RA (6.3-3.7 applications per animal). The mean power output was $25.7-6.5 \mathrm{~W}$, temperature $55.8-5.4^{\circ} \mathrm{C}$, impedance 91.1-5.5 $\Omega$, and duration 52.2-29.2 seconds (see Online Supplemental Table 1).

Concomitant recording of the EGM and atrial wall image allowing measurement of myocardial thickness before and after RF application was obtained in all but 2 sites where the catheter position changed during RF application.

Late morphological changes (5-min post-RF) with a concomitant EGM were observed in 26 sites, and interpretable images of myocardial thickness were obtained in all but one site.

\section{Validation of IVUS accuracy}

A total of 19 tissue samples were obtained from RF application sites in group 2 to be correlated with echographic images. Histological and echographic measurements of wall thickness could be correlated in 14 sites. In 2 sites, steam pops occurring during RF application prevented accurate measurement on IVUS images. In 3 tissue samples, interpretation was hampered by inadequate preparation. Histological and echographic measurements of atrial wall thickness were significantly correlated $(r=0.71 ; P=.004)$ (Figure 2).

\section{Description of RF effect on the atrial wall}

IVUS allowed an accurate visualization of the atrial wall before, during, and after RF application. During RF application, image was disturbed by electrical noise but was still interpretable. Before and after RF application, the tip of the 


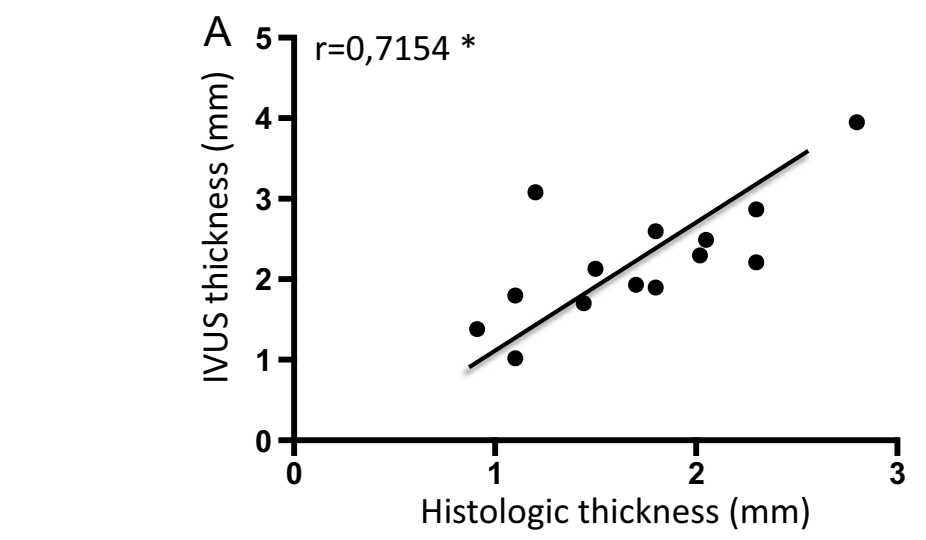

B
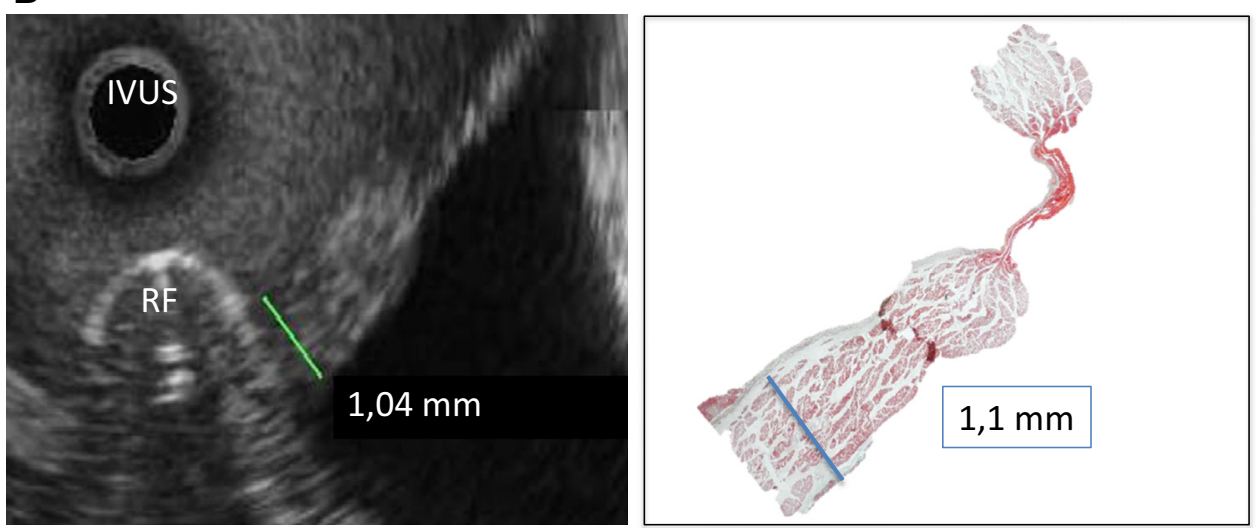

Figure 2 Comparison between B-mode intravascular ultrasound (IVUS) image and histology. A: Correlation between echographic and histological thickness measurement. ${ }^{*} P=.004$. B: Representative example.

RF catheter generated an acoustic shadow, but the atrial wall could be clearly seen right off it, and a slight rotation of the catheter allowed visualization of the RF application zone itself.

\section{Changes in atrial wall thickness and morphology}

In the early seconds after RF application, we observed significant changes in atrial wall morphology:

1. Atrial wall thickening occurred usually a few seconds after some RF applications, whereas significant changes in the EGM could be observed without a major change in wall thickness in the others (Figure 3).

2. Fluid accumulation, either intramyocardial or epicardial, was detected by IVUS imaging in 34 of 113 runs. In 16 of these 34 runs, a hypoechoic area developed within myocardial layers (Figure 4). In most cases, these changes could be seen during RF application after a few seconds. Epicardial fluid accumulation occurred a few minutes later but could sometimes be seen before RF application if other sites had been treated previously in the vicinity.

Other unusual but significant changes in atrial wall morphology could also be recorded:

1. Probable intramural bleeding: Local change in intramural echogenicity with a dramatic and rapid change in wall thickness and shape (Figure 4 and online supplemental material material \#1).
2. Steam pop: Two long (120-second) RF applications were performed with temperature limitation turned off in order to create a clinical steam pop. Without any precursor signs except for the usual change in atrial wall thickness, steam pop produced brutal surface echogenicity changes, probably corresponding to blood coagulation. Histological analysis revealed important disruption of atrial wall architecture and intramural edema (Figure 5 and additional material 2). Of note, we could see neither morphological changes nor microbubbles that could predict the steam pop as mentioned in other studies. ${ }^{25,26}$

3. Thrombus formation on the RF catheter tip: In all RF applications with this nonirrigated catheter, only 1 thrombus was noted, together with intramural bleeding, after prolonged and repeated RF application (Figure 5 and additional material \#3).

\section{Catheter contact and respiratory movements}

Coupled IVUS and RF catheters offered useful information about the contact between the RF catheter tip and the atrial wall. Respiratory changes and subsequent electrical potential shift could be recorded, demonstrating that contact surface and EGM amplitude could vary significantly along the respiratory cycle. IVUS could also detect small changes in contact surface induced by respiratory movement, slight rotations of the catheter, or application in anfractuous surfaces such as the coronary sinus ostium, the crista terminalis, or the cavotricuspid isthmus. 


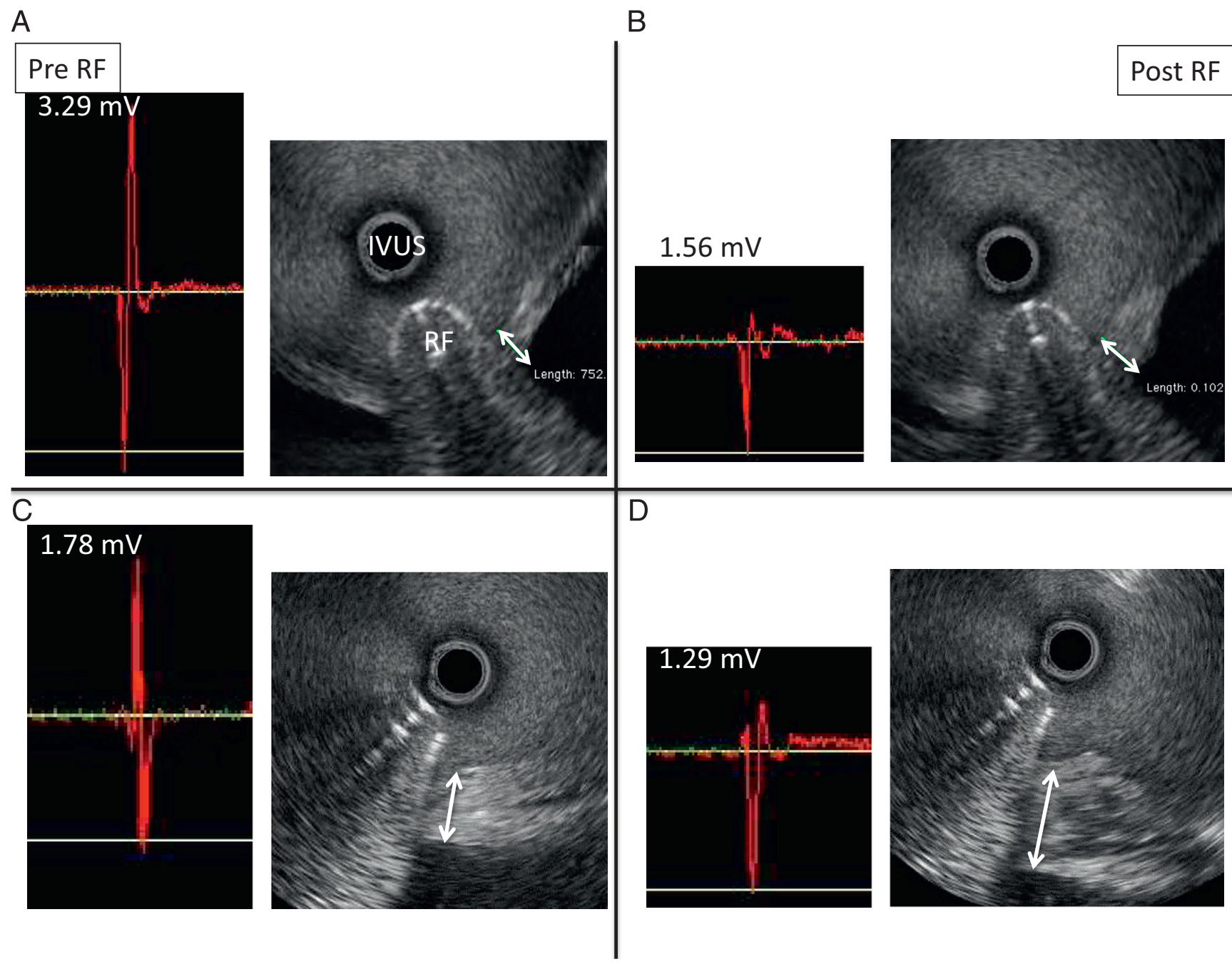

Figure 3 Electrogram (EGM) vs thickness relationship. A and B: significant EGM change without significant increase in thickness before and after RF application. C and D: Significant EGM change with dramatic thickening of the atrial wall. The arrow indicates the atrial wall. The intravascular ultrasound image shows the echo probe and the tip of the radiofrequency catheter, with the acoustic shadow generated beyond.

\section{IVUS imaging and criteria of transmural lesion}

The maximal positive deflection ( $\mathrm{R}$ wave) decreased from $1.17-0.66$ to $0.36-0.22 \mathrm{mV}(62 \% \pm 25 \% ; P<.0001)$ after $\mathrm{RF}$ application. The total voltage amplitude decreased from $2.20-1.11$ to $0.99-0.62 \mathrm{mV}(50 \% \pm 27 \% ; P<.0001)$. The maximal $\mathrm{R}$ wave and total voltage amplitude, 5 minutes after RF application, were $0.36-0.31$ and $0.85-0.65 \mathrm{~V}$, respectively (ie, not significantly different from those measured immediately after RF application) (Table 1).

The atrial wall thickness changed from $1.34-0.53 \mathrm{~mm}$ before RF application to $1.93-0.80 \mathrm{~mm}$ after RF application $(48 \% \pm 47 \% ; P<.0001)$. After 5 minutes, it was $1.84-0.78$ $\mathrm{mm}$, that is, not significantly different from that measured immediately after RF application (Table 1). There was a significant correlation between wall thickening and either Rwave amplitude decrease $(r=0.36)$ or total voltage amplitude decrease $(r=0.43)(P<.05$ for each) (Figure 6$)$.

In 40 of $56 \mathrm{RF}$ applications (71.4\%), a greater than $20 \%$ increase in wall thickness occurred, but EGM amplitude decrease $(1.35 \pm 1.32)$ was not significantly different from that in the other 16 cases $(0.77 \pm 0.58 \mathrm{mV})$. In 11 cases, RF application led to a greater than $80 \%$ decrease in EGM amplitude and atrial wall thickening was greater (1.04 \pm $0.47 \mathrm{~mm})$ than that in the 51 other cases $(0.51 \pm 0.47 \mathrm{~mm}$; $P=.0047)$. However, the percentage of wall thickening was not significantly different $(76 \% \pm 54 \%$ vs $45 \% \pm 45 \%$; $P=.07)$.

In the 19 tissue samples available in group 2, all could be analyzed for the transmural extent. In 5, the lesions were nontransmural (Figure 6). RF application duration was longer in transmural than in nontransmural lesions. EGM changes were nonsignificantly greater in the transmural group, and there was no difference in wall thickening (Table 2).

\section{Discussion}

We could observe, for the first time, the effects of RF application on the atrial wall, by coupling the IVUS probe to the RF catheter, in a porcine model. Several authors ${ }^{20,27,28}$ 


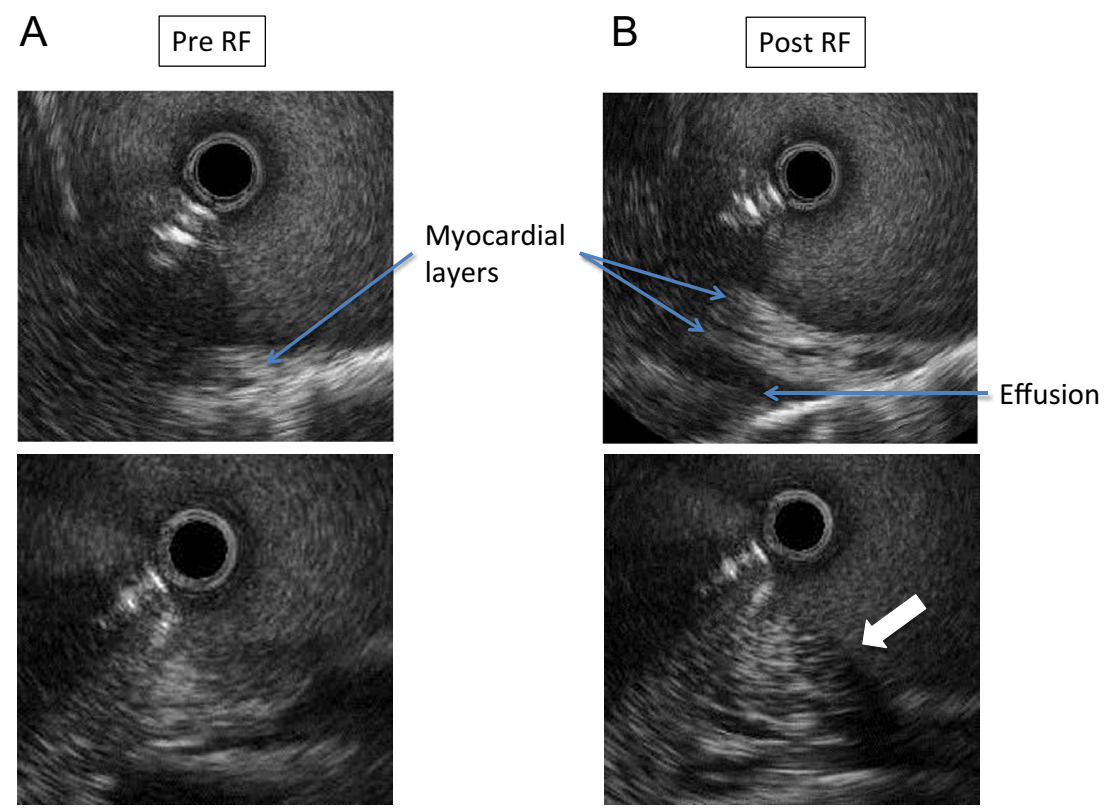

Figure 4 Morphological changes during radiofrequency (RF) application. A: Epicardial and intramyocardial effusion visible after RF application (compared to before RF application on top left). Catheter has been slightly turned clockwise to allow clear visualization of the RF catheter contact area. B: Dramatic changes in atrial wall structure: probable intraparietal hematoma (white arrow). Myocardial layers separated by effusion is visible under the thrombus. On the left panel: Pre-RF run is recorded in an area of multiple RF applications. An epicardial effusion is already visible (see video file in additional material \#1).

reported their experience of monitoring RF with echocardiographic guidance, but transesophageal or even intracardiac imaging does not yield a sufficiently high resolution to assess morphological changes in the atrial wall. Furthermore, assessing the precise location of the catheter tip and of the ablation zone is quite difficult when the echographic probe is not integrated with the ablation catheter. Wright et $\mathrm{al}^{11}$ recently described M-mode imaging of RF ablation by using a laboratory-made catheter in a sheep model, but a relatively small number of RF applications were performed
A
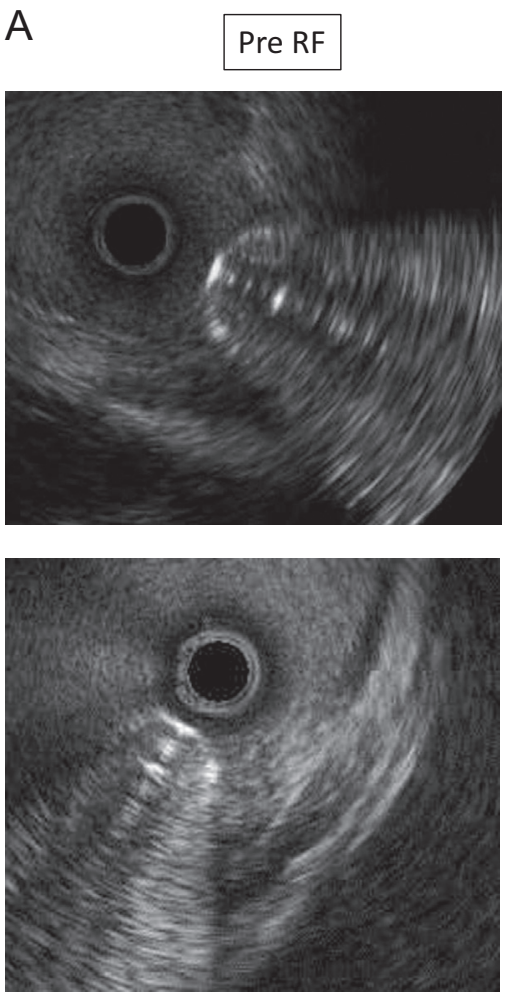

B

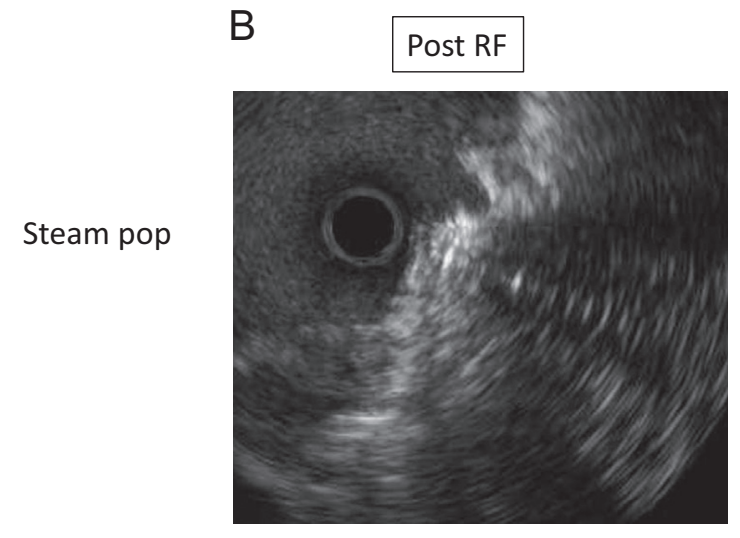

Thrombus on Catheter tip

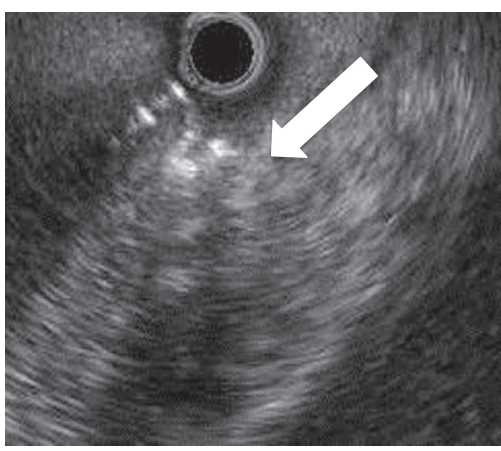

Figure 5 Morphological changes during radiofrequency (RF) application. A: Steam pop: Morphological changes before (top left) and immediately after (top right) RF application. After a dramatic increase in atrial wall thickness, RF catheter is suddenly embedded by thrombus and/or carbonization. B: Thrombus formation on the tip of the RF catheter: After significant changes in both electrogram and atrial wall thickness, formation of a mobile hyperechogenic thrombus on the tip of the RF catheter at the end of RF application (see video files in additional material \#2 and 3). 
Table 1 Results of ablation on electrogram and atrial wall thickness

\begin{tabular}{llll}
\hline EGM $(\mathrm{mV}) /$ Thickness $(\mathrm{mm})$ & Before RF $(\mathrm{n}=57)$ & After RF $(\mathrm{n}=57)$ & $5 \mathrm{~min}$ after RF $(\mathrm{n}=26)$ \\
\hline R-wave max $(\mathrm{mV})$ & $1.17 \pm 0.66$ & $0.36 \pm 0.22^{*}$ & $0.36 \pm 0.31$ \\
Total amplitude voltage $(\mathrm{mV})$ & $2.20 \pm 1.11$ & $0.99 \pm 0.62^{*}$ & $0.85 \pm 0.65$ \\
Atrial wall thickness $(\mathrm{mm})$ & $1.34 \pm 0.53$ & $1.93 \pm 0.80^{*}$ & $1.84 \pm 0.78$ \\
\hline
\end{tabular}

$\mathrm{RF}=$ radiofrequency

${ }^{*} P<.0001$ compared to before RF application.

endocardially in the atrium (only 1 of 11 endocardial lesions was interpretable). ${ }^{11}$ With its small diameter $(3.5 \mathrm{~F})$, the 40 $\mathrm{MHz}$ IVUS probe could be coupled to the RF catheter and used during RF application to provide high-resolution Bmode images.

Although IVUS accuracy has been largely demonstrated for the assessment of coronary artery lesions, its use to provide interpretable images of the atrial wall in the presence of the RF catheter and during RF application had not been tested so far. We could obtain interpretable images, and the IVUS probe sustained several RF applications without apparent damage. It allowed real-time diagnosis of intramural edema, microthrombus formation on the catheter tip or on the endocardial surface of the atrial wall, and epicardial effusion. Myocardium could also be easily differentiated from endo- or epicardium.

\section{Imaging of the atrial wall during RF application}

We could see that RF application could lead to dramatic and rapid changes in atrial wall thickness and shape, which confirm results reported by previous authors ${ }^{20,21}$ who used intracardiac echography not coupled to the RF catheter. Wall thickness increase appeared to be mostly due to edema or microscopic intramural hemorrhage, as revealed by histological analysis. Formation of intramural or intracavitary thrombus, development of an intramural or epicardic hypoechoic area suggesting effusion, or visualization of an adjacent anatomical structure (eg, aorta and left atrium) beyond the atrial wall could be of interest for clinical practice, as it should lead the operator to change his or her strategy and especially avoid repetitive RF applications when the EGM does not exhibit a significant decrease. Real-time imaging of RFinduced atrial tissue morphological changes allows illustrating the concept of "too much ablation." In the current clinical practice, as far as impedance and temperature are stable, there is no safety end point to stop RF application. EGM disappearance is the most widely used efficiency end point, but echographic imaging could provide a new safety end point to decide when to stop RF application.

However, there were some limitations in RF imaging by the IVUS probe. IVUS accuracy exhibits a good, but not perfect, correlation with histological samples. This could be explained by the measurement of tissue sample thickness that depends on the angle section, whereas in vivo thickness

\section{A}

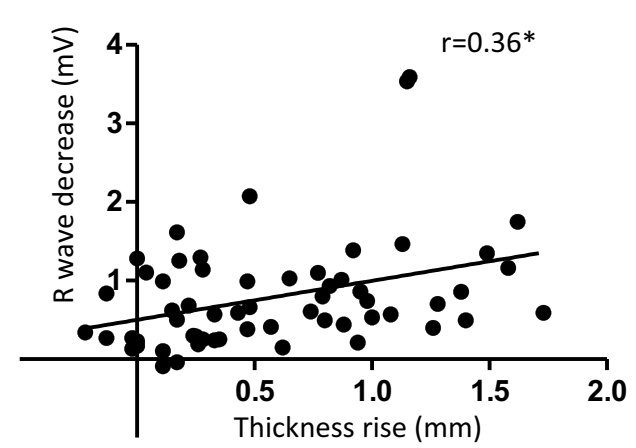

$\mathrm{B}$

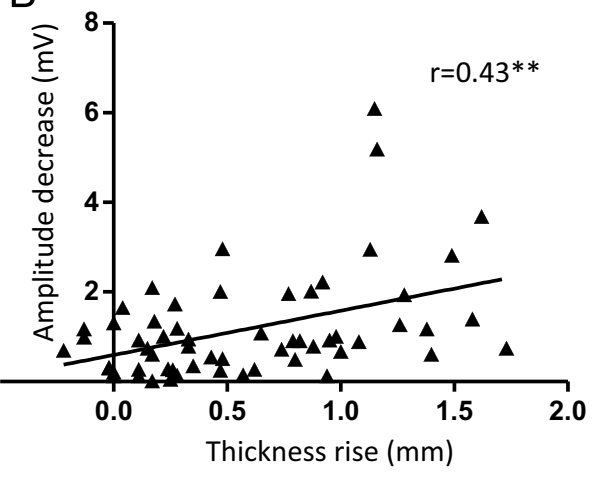

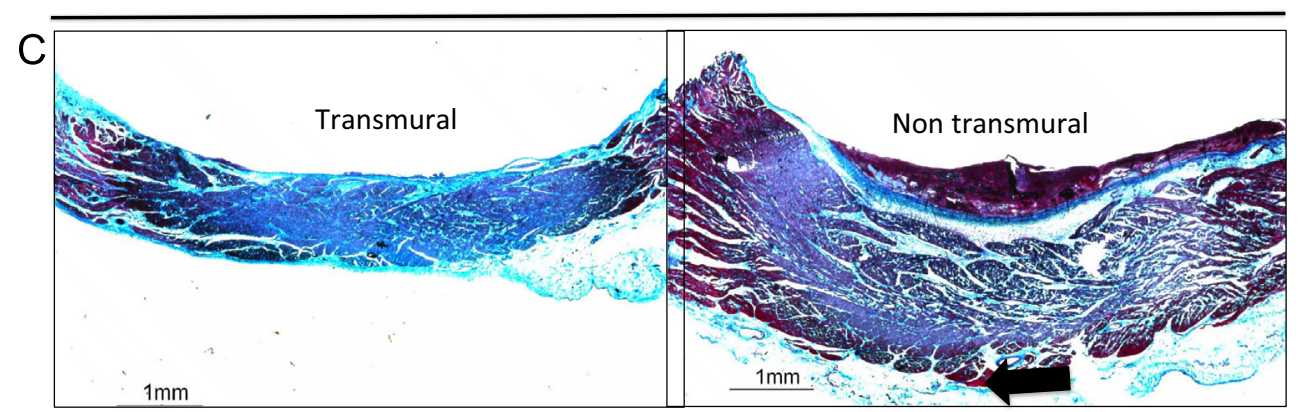

Figure 6 Transmural extent. A: Correlation between thickness increase and R-wave amplitude decrease. ${ }^{*} P=.0052$. B: Correlation between thickness increase and total voltage amplitude decrease. ${ }^{* * *} P=.0007$. C: Representative example of histologically transmural (left) and nontransmural (right) lesion (black arrow: epicardial gap). 
Table 2 Comparative results of histologically nontransmural and transmural RF applications

\begin{tabular}{lccc}
\hline RF applications & Nontransmural $(\mathrm{n}=5)$ & Transmural $(\mathrm{n}=14)$ & $P$ \\
\hline Power $(\mathrm{W})$ & $27 \pm 1.4$ & $25.5 \pm 4.5$ & $\mathrm{NS}$ \\
Temperature $\left({ }^{\circ} \mathrm{C}\right)$ & $57 \pm 1.7$ & $55 \pm 4$ & $\mathrm{NS}$ \\
Impedance $(\Omega)$ & $90.7 \pm 5.4$ & $93 \pm 5.67$ & $\mathrm{NS}$ \\
Duration $(\mathrm{s})$ & $54.2 \pm 39.2$ & $93.07 \pm 28.8$ & .045 \\
R-wave decrease $(\mathrm{mV})$ & $0.71 \pm 0.6(65 \pm 28)$ & $1.28 \pm 1.1(70 \pm 21)$ & $\mathrm{NS}$ \\
Amplitude decrease $(\mathrm{mV})$ & $1.17 \pm 1.4(48 \pm 27)$ & $1.98 \pm 1.7(64 \pm 26)$ & $\mathrm{NS}$ \\
Thickness increase $(\mathrm{mm})$ & $0.98 \pm 0.6(87 \pm 70)$ & $0.85 \pm 0.5(81 \pm 65)$ & $\mathrm{NS}$ \\
\hline
\end{tabular}

Values are expressed as mean \pm SD.

$\mathrm{NS}=$ not significant; $\mathrm{RF}=$ radiofrequency.

measurement may vary with the angle of view and the contact force applied. IVUS imaging is easily interpretable on plane surfaces such as the posterior right atrial wall. Nevertheless, in locations with trabeculation or large motion amplitude, interpretation may prove much more difficult.

The tip of the RF catheter creates an acoustic shadow. Therefore, only the margin of the treated area can be directly monitored during RF application. Nevertheless, a slight rotation of the catheter allows visualization of the exact site of treatment immediately after RF application.

\section{Assessment of the transmural extent of RF-induced lesions}

We were able to monitor RF-induced changes in the atrial wall together with electrical changes that are the current accepted criteria to monitor RF efficiency in the catheterization laboratory, but we did not find any correlation between them. Some RF applications could lead to the disappearance of electrical activity without any significant change in atrial wall morphology, whereas some other could lead to dramatic changes without any significant electrical change. Unlike Wright et al, ${ }^{11}$ we could not find any significant, easily interpretable, predictive change in atrial wall echogenicity during the 64 runs we recorded. One explanation could be that these authors used a laboratory-made M-mode catheter, while we used a commercially available B-mode catheter. Moreover, as mentioned above, B-mode images were disturbed by electrical noise during RF application. Although images were still interpretable, subjective interpretation of echogenicity would have been hazardous. Therefore, we focused on the more objective and reproducible thickness measurement and its correlation with the transmural extent.

Assessing the transmural extent of RF-induced lesion is of great interest in the clinical practice. Recently, contact force catheters have been introduced to provide this precious additional information and are now widely used. ${ }^{7}$ They allow better efficiency of RF lesion, leading to faster procedure and less RF applications. ${ }^{29,30}$ Some authors have proposed algorithms to assess the probability of transmural lesion by using a composite index of delivered power, time, and contact force..$^{10,31}$ However, direct monitoring of RF-induced lesions and their transmural extent is still not available.

As emphasized recently by Ikeda et $\mathrm{al}^{32}{ }^{32}$ contact force influences lesion size, but neither contact force nor EGM changes are good predictors of the transmural extent.
In our study, we did not find any echographic criteria of transmural lesions, but we did not evaluate criteria other than acute changes in wall thickness. Following up lesions for a few weeks after RF application and/or new image processing techniques such as thermal strain imaging could be of interest and mandate further investigation.

Some issues remain to be solved before coupled RF and IVUS catheters can be introduced into the clinical practice. We did not perform ablation procedures in the left atrium because of technical issues and the size of animals. We did not use irrigated RF catheters. Imaging the exact site of RFcatheter contact would be useful but technically challenging. Nevertheless, we are confident that a combined RF and IVUS catheter would be suitable for clinical use for RF ablation of atrial fibrillation or ventricular tachycardia.

\section{Conclusion}

An IVUS probe coupled to an RF catheter can provide relevant real-time imaging of the atrial wall during ablation. Although thickness change does not appear to be a good predictor of the transmural extent, direct visualization and monitoring of RF application may provide new information to guide and secure RF ablation.

\section{Acknowledgments}

We thank Xavier Brunet, MD, Clement Lonjon, MD, and Abdelkader Zedira, MD, for their precious help during experiments. We thank Michel Dauzat, MD, PhD, and Lionel Beck, $\mathrm{MD}, \mathrm{PhD}$, for their precious remarks and advice on the article.

\section{Appendix}

\section{Supplementary data}

Supplementary data associated with this article can be found in the online version at http://dx.doi.org/10.1016/j.hrthm. 2015.04.012.

\section{References}

1. Lavergne T, Guize L, Heuzey JYL, Carcone P, Geslin J, Cousin MT. Closedchest atrioventricular junction ablation by high-frequency energy transcatheter desiccation. Lancet 1986;2:858-859.

2. Huang SK, Bharati S, Graham AR, Lev M, Marcus FI, Odell RC. Closed chest catheter desiccation of the atrioventricular junction using radiofrequency energy - a new method of catheter ablation. J Am Coll Cardiol 1987;9:349-358.

3. Haines DE, Verow AF. Observations on electrode-tissue interface temperature and effect on electrical impedance during radiofrequency ablation of ventricular myocardium. Circulation 1990;82:1034-1038. 
4. Hartung WM, Burton ME, Deam AG, Walter PF, McTeague K, Langberg JJ. Estimation of temperature during radiofrequency catheter ablation using impedance measurements. Pacing Clin Electrophysiol 1995;18:2017-2021.

5. Wittkampf FH, Hauer RN, de Medina EOR. Control of radiofrequency lesion size by power regulation. Circulation 1989;80:962-968.

6. Ouyang F, Antz M, Ernst S, et al. Recovered pulmonary vein conduction as a dominant factor for recurrent atrial tachyarrhythmias after complete circular isolation of the pulmonary veins: lessons from double Lasso technique. Circulation 2005;111:127-135.

7. Yokoyama K, Nakagawa H, Shah DC, Lambert H, Leo G, Aeby N, Ikeda A, Pitha JV, Sharma T, Lazzara R, Jackman WM. Novel contact force sensor incorporated in irrigated radiofrequency ablation catheter predicts lesion size and incidence of steam pop and thrombus. Circ Arrhythm Electrophysiol 2008;1:354-362.

8. Shah DC, Lambert H, Nakagawa H, Langenkamp A, Aeby N, Leo G. Area under the real-time contact force curve (force-time integral) predicts radiofrequency lesion size in an in vitro contractile model. J Cardiovasc Electrophysiol 2010;21:1038-1043.

9. Reddy VY, Shah D, Kautzner J, et al. The relationship between contact force and clinical outcome during radiofrequency catheter ablation of atrial fibrillation in the TOCCATA study. Heart Rhythm 2012;9:1789-1795.

10. Neuzil P, Reddy VY, Kautzner J, Petru J, Wichterle D, Shah D, Lambert H, Yulzari A, Wissner E, Kuck K-H. Electrical reconnection after pulmonary vein isolation is contingent on contact force during initial treatment: results from the EFFICAS I study. Circ Arrhythm Electrophysiol 2013;6:327-333.

11. Wright M, Harks E, Deladi S, Suijver F, Barley M, van Dusschoten A, Fokkenrood S, Zuo F, Sacher F, Hocini M, Haïssaguerre M, Jaïs P. Real-time lesion assessment using a novel combined ultrasound and radiofrequency ablation catheter. Heart Rhythm 2011;8:304-312.

12. Eyerly SA, Bahnson TD, Koontz JI, Bradway DP, Dumont DM, Trahey GE, Wolf PD. Intracardiac acoustic radiation force impulse imaging: a novel imaging method for intraprocedural evaluation of radiofrequency ablation lesions. Heart Rhythm 2012;9:1855-1862.

13. Dana N, Di Biase L, Natale A, Emelianov S, Bouchard R. In vitro photoacoustic visualization of myocardial ablation lesions. Heart Rhythm 2014;11:150-157.

14. de Senneville BD, Roujol S, Jaïs P, Moonen CTW, Herigault G, Quesson B. Feasibility of fast MR-thermometry during cardiac radiofrequency ablation. NMR Biomed 2012;25:556-562.

15. Kolandaivelu A, Zviman MM, Castro V, Lardo AC, Berger RD, Halperin HR. Noninvasive assessment of tissue heating during cardiac radiofrequency ablation using MRI thermography. Circ Arrhythm Electrophysiol 2010;3:521-529.

16. Fleming CP, Quan KJ, Wang H, Amit G, Rollins AM. In vitro characterization of cardiac radiofrequency ablation lesions using optical coherence tomography. Opt Express 2010;18:3079-3092.

17. Matsumoto K, Yamamoto T, Saitou J, Uchida M, Tamura T, Imafuku H, Shindou A, Kaneko K, Asano Y, Dohi Y. Use of biplane transesophageal echocardiographic guide in radiofrequency catheter ablation of Wolff-Parkinson-White syndrome with left side Kent bundle. Jpn Circ J 1993;57:832-836.

18. Chu E, Kalman JM, Kwasman MA, Jue JC, Fitzgerald PJ, Epstein LM, Schiller NB, Yock PG, Lesh MD. Intracardiac echocardiography during radiofrequency catheter ablation of cardiac arrhythmias in humans. J Am Coll Cardiol 1994;24:1351-1357.

19. Raczka F, Granier M, Cung TT, Davy JM. Intracardiac thrombus: a good indication of ultrasound image integration system (Cartosound) for radiofrequency ablation. Europace 2010;12:591-592.
20. Ren JF, Callans DJ, Schwartzman D, Michele JJ, Marchlinski FE. Changes in local wall thickness correlate with pathologic lesion size following radiofrequency catheter ablation: an intracardiac echocardiographic imaging study. Echocardiography 2001;18:503-507.

21. Weerasooriya R, Jaïs P, Sanders P, Scavée C, Hsu L-F, Hocini M, Clementy J, Haïssaguerre M. Images in cardiovascular medicine: early appearance of an edematous tissue reaction during left atrial linear ablation using intracardiac echo imaging. Circulation 2003;108:e80.

22. Isner JM, Rosenfield K, Losordo DW, Rose L, Langevin RE Jr, Razvi S, Kosowsky BD. Combination balloon-ultrasound imaging catheter for percutaneous transluminal angioplasty: validation of imaging, analysis of recoil, and identification of plaque fracture. Circulation 1991;84:739-754.

23. Schwartzman D, Michele JJ, Trankiem CT, Ren JF. Electrogram-guided radiofrequency catheter ablation of atrial tissue comparison with thermometry-guide ablation: comparison with thermometry-guide ablation. J Interv Card Electrophysiol 2001;5:253-266.

24. Otomo K, Uno K, Fujiwara H, Isobe M, Iesaka Y. Local unipolar and bipolar electrogram criteria for evaluating the transmurality of atrial ablation lesions at different catheter orientations relative to the endocardial surface. Heart Rhythm 2010;7:1291-1300.

25. Wright M, Harks E, Deladi S, Fokkenrood S, Zuo F, Van Dusschoten A, Kolen AF, Belt H, Sacher F, Hocini M, Haïssaguerre M, Jaïs P. Visualizing intramyocardial steam formation with a radiofrequency ablation catheter incorporating near-field ultrasound. J Cardiovasc Electrophysiol 2013;24:1403-1409.

26. Koruth JS, Dukkipati S, Gangireddy S, McCarthy J, Spencer D, Weinberg AD, Miller MA, D'Avila A, Reddy VY. Occurrence of steam pops during irrigated RF ablation: novel insights from microwave radiometry. J Cardiovasc Electrophysiol 2013;24:1271-1277.

27. Olgin JE, Kalman JM, Chin M, Stillson C, Maguire M, Ursel P, Lesh MD. Electrophysiological effects of long, linear atrial lesions placed under intracardiac ultrasound guidance. Circulation 1997;96:2715-2721.

28. Doi A, Takagi M, Toda I, Teragaki M, Yoshiyama M, Takeuchi K, Yoshikawa J Real time quantification of low temperature radiofrequency ablation lesion size using phased array intracardiac echocardiography in the canine model: comparison of two dimensional images with pathological lesion characteristics. Heart 2003;89:923-927.

29. Andrade JG, Monir G, Pollak SJ, et al. Pulmonary vein isolation using "contact force" ablation: the effect on dormant conduction and long-term freedom from recurrent AF. A prospective study. Heart Rhythm 2014;11:1919-1924.

30. Kimura M, Sasaki S, Owada S, Horiuchi D, Sasaki K, Itoh T, Ishida Y, Kinjo T, Tomita H, Okumura K. Comparison of lesion formation between contact forceguided and non-guided circumferential pulmonary vein isolation: a prospective, randomized study. Heart Rhythm 2014;11:984-991.

31. Squara F, Latcu DG, Massaad Y, Mahjoub M, Bun S-S, Saoudi N. Contact force and force-time integral in atrial radiofrequency ablation predict transmurality of lesions. Europace 2014;16:660-667.

32. Ikeda A, Nakagawa H, Lambert H, Shah DC, Fonck E, Yulzari A, Sharma T, Pitha JV, Lazzara R, Jackman WM. Relationship between catheter contact force and radiofrequency lesion size and incidence of steam pop in the beating canine heart: electrogram amplitude, impedance, and electrode temperature are poor predictors of electrode-tissue contact force and lesion size. Circ Arrhythm Electrophysiol 2014;7:1174-1180.

\section{CLINICAL PERSPECTIVES}

High-resolution real-time B-mode imaging of the atrial wall during radiofrequency (RF) ablation should help assessing the characteristics of RF-induced lesions, detecting side effects or complications, and understanding the relationship between morphological changes and functional results. Our preliminary results in a porcine model demonstrate the interest of developing coupled echographic and RF catheters, together with innovative image and signal processing tools, thus providing interventional cardiologists with a system that may be used for the adaptive setting of RF parameters and for monitoring results in the clinical routine. 